\title{
Genetic Algorithm and Fisher Discriminant Analysis based Wavelet Thresholding for Speckle Noise Filtering in Ultrasound Images
}

\author{
Shisir Mia \\ Department of Computer Science \\ and Engineering, \\ Mawlana Bhashani Science and \\ Technology University, Santosh, \\ Tangail-1902, Bangladesh
}

\author{
Md. Mahfuz Reza \\ Department of Computer Science \\ and Engineering, \\ Mawlana Bhashani Science and \\ Technology University, Santosh, \\ Tangail-1902, Bangladesh
}

\author{
Mohammad Motiur Rahman \\ Department of Computer Science \\ and Engineering, \\ Mawlana Bhashani Science and \\ Technology University, Santosh, \\ Tangail-1902, Bangladesh
}

\begin{abstract}
Speckle noise is a significant property of medical ultrasound imaging, and it typically degrades the resolution and contrast of images, sinking the diagnostic importance of the imaging modality. As a consequence, filtering speckle noise in the ultrasound images is a critical step for further analysis by the medical experts. In this paper, a speckle noise filtering technique have been suggested via wavelet thresholding for denosing ultrasound images. For each wavelet coefficient, in the first step, two optimal threshold parameters are estimated through the genetic algorithm and fisher discriminant analysis respectively. In the second step, thresholding of wavelet coefficient is performed by both threshold parameters. Finally, thresholded coefficient which corresponds to lowest mean square error is selected for obtaining the denoised ultrasound image. Results show that, the proposed technique outperforms different existing denoising techniques.
\end{abstract}

\section{General Terms}

Biomedical Image Processing; Computer Vision

\section{Keywords}

Genetic Algorithm; Fisher Discriminant Analysis; Speckle Noise; Ultrasound Image; Wavelet Transform

\section{INTRODUCTION}

Noise filtering in images is the most critical and fundamental tasks in image processing and computer vision. The aim of the noise filtering technique is to diminish noise while preserving essential image details or features. Speckle is a specific form of multiplicative noise seen in images obtained via the coherent imaging systems like ultrasound. It tends to degrade the ultrasound image resolution and contrast, thus potentially obliterating some valuable and significant diagnostic details. Wavelet based speckle noise filtering has been the focus of numerous research work in recent decades since wavelet provides an appropriate basis for separating noise signal from image signal. The central idea of this method is to transform the noisy image into the wavelet domain, threshold the wavelet coefficients, and perform the inverse wavelet transformation. The key problem with the wavelet thresholding approach is to determine the proper threshold value, since a small threshold value would allow all noisy coefficients to move through and as an effect, the denoised signal can also appear to be noisy. A large threshold value, on the other indicator, causes a huge extent of coefficients to be zero, which results in a smooth signal while destroying information and causing blur and artifacts in the image. Many wavelet thresholding techniques i.e., hard thresholding, soft thresholding, VisuShrink, SureShrink, BayesShrink, and Bayes thresholding [1-10] for image denoising have shown improved performance in image processing.

Fisher Discriminant Analysis (FDA) and Genetic Algorithm (GA) were used to find out the proper threshold parameter to perform the thresholding of sub-band coefficients in wavelet domain for denosing ultrsasound images [11-12]. The authors have achieved the better outcomes for reducing noise in ultrasound images in their recommended work.

Although many research has been done to develop the method for filtering speckle noise in ultrasound images, no optimal method has yet been developed. An effective speckle noise filtering technique have been proposed through wavelet thresholding in which the threshold parameters are estimated through FDA and GA, and hence, the technique provides the promising efficacy for reducing speckle noise. The main contribution of this research are as follows:

i. Genetic Algorithm (GA) and Fisher Discriminant Analysis (FDA) are applied to estimate two optimal threshold parameters for effectively thresholding each and every noisy wavelet sub-band coefficient.

ii. Mean square error (MSE)-based comparison is used to select the finest filtered sub-band coefficient.

iii. The quantitative analysis and visual investigation are performed to demonstrate the encouraging efficiency of the proposed technique.

\section{THEORETICAL BACKGROUND}

\subsection{Speckle Noise Model}

Let's, $I(x, y)$ and $f(x, y)$ denote noisy and clean ultrasound images respectively. The speckle affected image can be defined as,

$I(x, y)=f(x, y) \times \eta_{m}(x, y)+\eta_{a}(x, y)$

Where, $\eta_{m}(x, y)$ and $\eta_{a}(x, y)$ are the corrupting speckle noise components. In most cases, the additive part of the speckle in ultrasound images has a smaller impact than the multiplicative component. Thus, ignoring the term $\eta_{a}(x, y)$, equation (1) can be rewritten as,

$I(x, y)=f(x, y) \times \eta_{m}(x, y)$ 
In order to alter the model of multiplicative noise into an additive one, logarithmic function is applied on together sides of equation (2).

\subsection{Wavelet Transform}

Wavelet transformation is performed using 2D scaling and wavelet functions. The scaled and translated basis functions are:

$$
\begin{aligned}
& f_{j, m, n}(x, y)=2^{j / 2} f\left(2^{j} x-m, 2^{j} y-n\right) \\
& \psi_{j, m, n}^{i}(x, y)=2^{j / 2} \psi^{i}\left(2^{j} x-m, 2^{j} y-n\right)
\end{aligned}
$$

Where $i=\{H, V, D\}$. The Discrete Wavelet Transform (DWT) of a function $f(x, y)$ of size $M \times N$ can be defined as,

$W_{f}\left(j_{0}, m, n\right)=\frac{1}{\sqrt{M N}} \sum_{x=0}^{M-1} \sum_{y=0}^{N-1} f(x, y) f_{j_{0}, m, n}(x, y)$

$W_{\psi}^{i}(j, m, n)=\frac{1}{\sqrt{M N}} \sum_{x=0}^{M-1} \sum_{y=0}^{N-1} f(x, y) \psi_{j, m, n}^{i}(x, y)$

Where $i=\{H, V, D\}$. By applying DWT on image, four subband coefficient values are obtained. Those sub-bands are Approximation and Detail, Detail includes horizontal, vertical, diagonal. The inverse operation is performed to get the previous data back. The Inverse Discrete Wavelet Transform (IDWT) is:

$f(x, y)=\frac{1}{\sqrt{M N}} \sum_{m} \sum_{n} W_{f}\left(j_{0}, m, n\right) f_{j_{0}, m, n}(x, y)+$
$\frac{1}{\sqrt{M N}} \sum_{i=H, V, D} \sum_{j=j_{0}}^{¥} \sum_{m} \sum_{n} W_{\psi}^{i}(j, m, n) \psi_{j, m, n}^{i}(x, y)$

(7)

Following the execution of IDWT, the data would return to its previous state and be rebuilt.

\section{PROPOSED TECHNIQUE}

In the initial step of the proposed scheme, 2 scale DWT is applied on input noisy image to get the approximation and details. Then for each sub-band coefficient, GA and FDA are used to estimate the two threshold parameters. Afterwards, hard thresholding operation is performed on the sub-band by both threshold parameters and subsequently, the mean square errors (MSEs) are computed. If the MSE is lowest for the thresholded sub-band with respect to the GA based threshold value then this thresholded sub-band is chosen for reconstructing the de-speckled ultrasound image, otherwise thresholded sub-band with respect to FDA based threshold parameter is selected. Thus, all the thresholded sub-band corresponds to lowest MSE are gotten. Finally, denoised image is obtained by way of applying the inverse operation of DWT. Fig 1 shows the block diagram of the proposed technique.

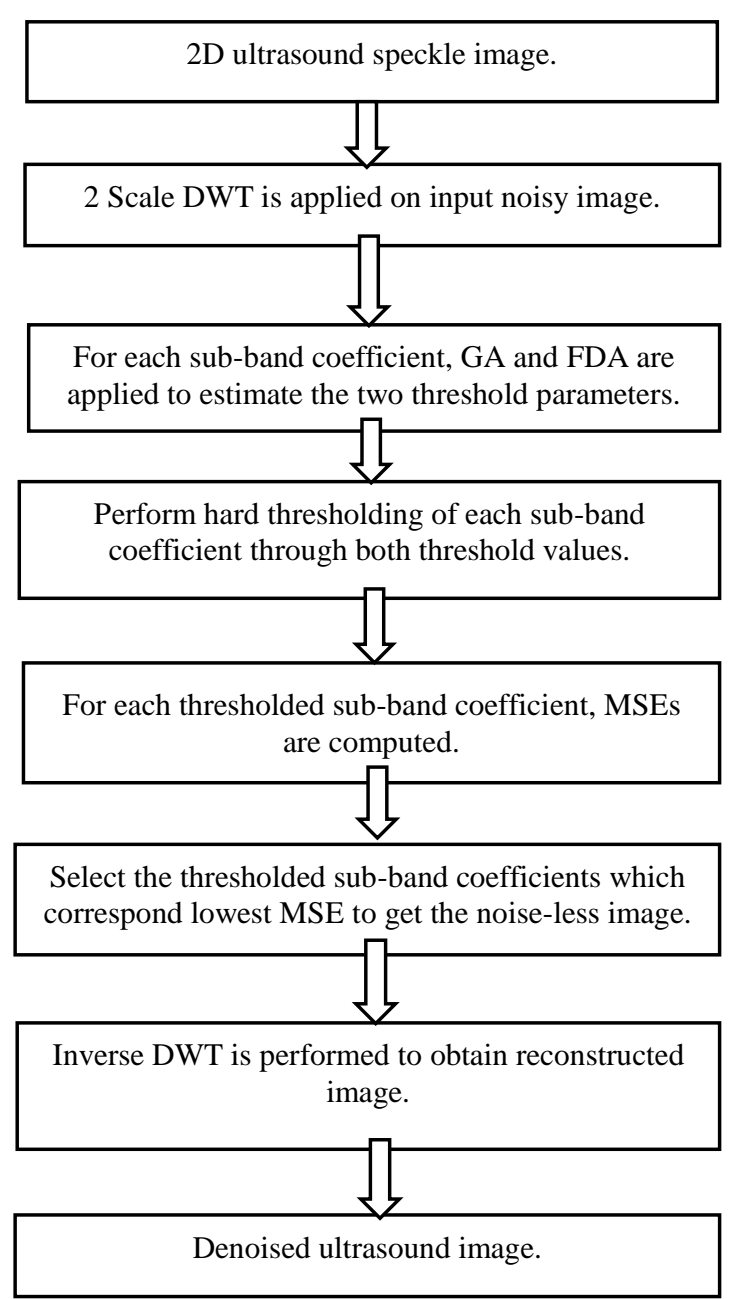

Fig 1: Block diagram of the proposed technique

\subsection{GA Based Threshold Parameter}

Genetic algorithm (GA) is a stochastic randomized search algorithm that is motivated through biological evolutionary process and driven by natural genetic systems. The fitness of all individuals is determined after the initial populations of individuals are encoded at random. The fittest individuals move through the selection, crossover, and mutation stages of the GA iteratively before an appropriate solution is found.

Genetic algorithm begins searching for the proper threshold parameter through encoding the population randomly to filter the wavelet sub-band coefficients of noisy image. In this technique, the threshold parameter is represented by an encoded binary chromosome $P$ of eight bit length. The objective is to minimize the value of mean square error (MSE) given in equation (8), as a result this is the fitness function $f$ of each chromosome in the GA based technique to estimate optimal threshold parameter.

$$
M S E=\frac{1}{M \times N} \sum_{x=0}^{M-1} \sum_{y=0}^{N-1}\left(\xi(x, y)-\xi_{d}(x, y)\right)^{2}
$$

Where $M$ and $N$ are the dimensions of the wavelet sub-band coefficients respectively. The noisy sub-band coefficients and filtered sub-band coefficients are represented by $\xi$ and $\xi_{d}$ respectively.

The fittest chromosome of each generation is copied to the next without being involved in the crossover and mutation 
stages. Outside the population, one copy of the preeminent chromosome is saved. The worst chromosome is also selected in the current generation. If the worst chromosome is superior to the best from the previous generation, it survives; otherwise, the best chromosome from the previous generation takes its place. This type of genetic algorithm is referred to as Elitism model.

The fittest chromosomes were selected using binary tournament selection (BTS) [13] to create a mating pool of the same size as the population. Two chromosomes are nominated randomly from the population, and the better one is copied into the mating pool of the next generation until the pool is empty. The tie is resolved at random.

Crossover is a high-probability operation that occurs each time between two chromosomes which are selected randomly. The proposed technique employs the uniform crossover method. For the pool size of $n$, it is repeated $n / 2$ size. To begin, two chromosomes are carefully chosen at random from the pool. A binary mask with the same size of the chromosome is produced at random. The technique involves checking for the mask bit value, and if it is one, bitwise swapping the bit values of the two chromosomes. The bit position is not swapped if this is not achieved.

After crossover every offspring undergoes mutation which is also a probabilistic operation. Mutating a bit means just changing 0 to 1 otherwise 1 to 0 . It is performed with very low probability.

The parameters of this technique is listed below:

- Population size: [15-20]

- Chromosome length is fixed with 8

- Crossover probability $\mu_{c}=[0.5-0.9]$

- Mutation probability $\mu_{m}=[0.3-0.6]$

- Number of generation [15-20].

The threshold parameter estimation using GA starts with encoding a population. Say, it encodes a population of having $n$ chromosomes. The binary chromosome is transformed to decimal which is actually the threshold parameter. The threshold is applied to filter the wavelet sub-band coefficients of noisy images through hard thresholding. The hard thresholding approach is given as,

$T h_{\text {Hard }}= \begin{cases}Y & \text { if }\|Y\|>\lambda \\ 0 & \text { Otherwise }\end{cases}$

(9)

In hard thresholding, if the absolute value of the wavelet subband coefficient is less than the threshold parameter $\lambda$, then it is set to 0 , otherwise it remains unchanged [9].

Based on the mean square error (MSE) between noisy and filtered sub-band coefficients, the fitness is computed applying the new threshold for each chromosome. Next corresponding stages are repeated to go the next generation. After a number of iterations the optimal threshold parameter is obtained.

\subsection{FDA Based Threshold Parameter}

The DWT of an image $f(x, y)$ of size $M \times N$ is defined by,

$$
W_{\psi}^{i}(j, m, n)=\frac{1}{\sqrt{M N}} \sum_{x=0}^{M-1} \sum_{y=0}^{N-1} f(x, y) \psi_{j, m, n}^{i}(x, y)
$$

Where, $i=\{H, V, D\}$ indicates the horizontal, vertical and diagonal sub-band coefficients. Let, each coefficient is represented individually by $n_{i}$ and, the entire number of coefficients $N=n_{0}+n_{1}+n_{2}+\ldots+n_{M}$. The probability of each coefficient is calculated by the following equation:

$P_{i}=\frac{n_{i}}{N}$

Where, $\sum_{i=1}^{M} P_{i}=1$ and $P_{i} \geq 0$.

Assume that, the coefficients are divided into two classes $C_{1}$ and $C_{2}$, the set of coefficients with labels $[0,1,2, \ldots, L]$ belongs to class $C_{1}$ and rest of the coefficients belongs to class $C_{2}$ and, generally $C_{1}$ and $C_{2}$ corresponds to the object class and the background one or vice versa. Then, the probabilities of two classes are given by within as,

$W_{1}(L)=\sum_{i=0}^{L} P_{i}$

$W_{2}(L)=1-W_{1}(L)$

For the classes $C_{1}$ and $C_{2}$, the mean coefficients can be computed as,

$\mu_{1}=\sum_{i=0}^{L} \frac{i P_{i}}{W_{1}}$

$\mu_{2}=\sum_{i=L+1}^{M} \frac{i P_{i}}{W_{2}}$

Thus the corresponding class variances are calculated through the following equations:

$\sigma_{1}^{2}=\sum_{i=0}^{L} \frac{\left(i-\mu_{1}\right)^{2} P_{i}}{W_{1}}$

$\sigma_{2}^{2}=\sum_{i=L+1}^{M} \frac{\left(i-\mu_{2}\right)^{2} P_{i}}{W_{2}}$

The within-class variance can be defined as [14],

$\sigma_{W}^{2}=W_{1} \sigma_{1}^{2}+W_{2} \sigma_{2}^{2}$

Since, the Fisher Linear Discriminant (FLD) seeks the most efficient direction for discrimination by yielding the highest ratio of between-class scatter to within-class scatter. As a consequence, according to the FLD criteria, the following objective function is applied to determine the separability of the threshold at level $L$.

$\rho(L)=\frac{\left(\mu_{1}(L)-\mu_{2}(L)\right)^{2}}{\sigma_{W}^{2}}$

Through the equation (16), FDA threshold parameter $T$ can be computed [15]. The threshold $T$ can successfully distinguish two classes, but this parameter is not much effective for reducing the noise. Since the mean and variance of the speckle in an ultrasound image have a linear relationship, the proper threshold value can be estimated through applying the standard deviation and mean value of the wavelet sub-band coefficient. When the DWT of an image $f(x, y)$ is $\xi(x, y)$, and the image size is $P \times Q$, then the mean value of the subband coefficient of wavelet is defined as,

$\mu_{c}=\frac{1}{P \times Q} \sum \xi^{i}(x, y)$

Where $i=\{H, V, D\}$. The standard deviation of the sub-band coefficient of wavelet can be computed as,

$\sigma_{c}=\sqrt{\frac{\sum_{x=0}^{P-1} \sum_{y=0}^{Q-1}\left[\xi(x, y)-\mu_{c}\right]^{2}}{P \times Q}}$

Since, for the large FDA threshold value, huge amount of significant information of the image is damaged. Hence, for effective outcomes of speckle reduction, the following equation is proposed to estimate the optimal threshold parameter:

$T_{\text {optimal }}=\frac{T}{\sqrt{\left|\sigma_{c} / \mu_{c}\right|}}$ 
Where, $T$ denotes the FDA threshold parameter and $\frac{\sigma_{c}}{\mu_{c}}>0$.

\section{EVALUATION CRITERIA}

The performance of different speckle reducing techniques have been observed for decreasing the speckle noise through applying Signal-to-Noise Ratio (SNR), Peak Signal-to-Noise Ratio (PSNR) and, Image Quality Index (IMGQI) and, Edge Preservation Factor (EPF) [16-17]

Signal-to-Noise Ratio (SNR): The higher the SNR value, the better the noise reduction outcomes. The SNR can be calculated using the equation below:

$S N R=-10 \log 10\left[\frac{\sum_{x=1}^{M} \sum_{y=1}^{N}\left(I_{d}(x, y)-I(x, y)\right)^{2}}{\sum_{x=1}^{M} \sum_{y=1}^{N}\left(I_{d}(x, y)\right)^{2}}\right]$

Peak Signal-to-Noise Ratio (PSNR): The higher the PSNR value, the better the image is transformed, and the lower the value, the worse the image is transformed. The PSNR is a metric for determining image fidelity which indicates how closely the transformed image resembles the original. The following formula can be applied to calculate it:

$P S N R=10 \log 10\left(\frac{S^{2}}{M S E}\right)$

Where $S$ denotes the maximum intensity in the original image, and $M S E$ is the mean square error between the filtered image and the original image.

Image Quality Index (IMGQI): IMGQI can decide the degree of distortion in terms of loss of correlation. IMGQI has a dynamic range of -1 to 1 , with higher values indicating better image quality. The following equation is used to measure it:

$I M G Q I=\frac{4 \sigma_{I I_{d}} \overline{I_{d}}}{\left(\sigma_{I}^{2}+\sigma_{I_{d}}^{2}\right)\left(\bar{I}^{2}+\bar{I}_{d}^{2}\right)}$

In equation (20) and (22), $I$ and $I_{d}$ denote the original and filtered images respectively, $\sigma$ represents the standard deviation, $\bar{I}$ and $\overline{I_{d}}$ are the mean value of the original image and filtered image respectively, and $M \times N$ is the image size.

Edge Preservation Factor (EPF): The higher the EPF value, the greater the ability to preserve the edges. The following equation can be used to compute EPF:

$E P F=\frac{\sum(\Delta I-\overline{\Delta I})\left(\Delta I_{d}-\overline{\Delta I_{d}}\right)}{\sqrt{\sum(\Delta I-\overline{\Delta I})^{2}\left(\Delta I_{d}-\overline{\Delta I_{d}}\right)^{2}}}$

Where $\Delta I$ and $\Delta I_{d}$ are the high pass filtered versions of original image $I$ and filtered image $I_{d}$ respectively, obtained with a $3 \times 3$ pixel standard approximation of Laplacian operator.

\section{RESULTS AND DISCUSSION}

In order to ensure the efficiency of the proposed technique, Liver and Kidney ultrasound images of size $256 \times 256$ have been considered which have been corrupted by speckle noise of variance 0.004. The experimentation is performed on MATLAB 9.4.0.813654 (R2018a) in a Core i5 $1.60 \mathrm{GHz}$ and 8GB RAM laptop having Windows operating system. The proposed technique is compared with numerous thresholding approaches which have been shown by Table 1, 2 and Fig 2, 3 , and 4 respectively. The quantitative comparison of various thesholding techniques with the proposed scheme for reducing the speckle noise in Liver and Kidney ultrasound images is shown in Table 1 and 2 . According to the statistical measurement parameters such as SNR, PSNR, EPF and IMGIQ, the proposed technique provide better outcomes to reduce the speckle noise compared to other existing denoising techniques including FDA based thresholding method.

Table 1. Quantitative performance of the proposed technique including different thresholding approaches for denoising Liver ultrasound image

\begin{tabular}{|c|c|c|c|c|}
\hline Method & SNR & PSNR & EPF & IMGIQ \\
\hline $\begin{array}{c}\text { Hard } \\
\text { Thresholding }\end{array}$ & 10.68 & 26.41 & 0.33 & 0.57 \\
\hline $\begin{array}{c}\text { Soft } \\
\text { Thresholding }\end{array}$ & 10.82 & 26.58 & 0.36 & 0.58 \\
\hline $\begin{array}{c}\text { Bayesian } \\
\text { Thresholding }\end{array}$ & 11.10 & 27.03 & 0.46 & 0.58 \\
\hline $\begin{array}{c}\text { FDA Based } \\
\text { Thresholding }\end{array}$ & 11.38 & 27.45 & 0.55 & 0.57 \\
\hline $\begin{array}{c}\text { Proposed } \\
\text { Technique }\end{array}$ & $\mathbf{2 6 . 2 5}$ & $\mathbf{3 6 . 4 2}$ & $\mathbf{0 . 9 3}$ & $\mathbf{0 . 6 2}$ \\
\hline
\end{tabular}

Table 2. Quantitative performance of the proposed technique including different thresholding approaches for denoising Kidney ultrasound image

\begin{tabular}{|c|c|c|c|c|}
\hline Method & SNR & PSNR & EPF & IMGIQ \\
\hline $\begin{array}{c}\text { Hard } \\
\text { Thresholding }\end{array}$ & 11.85 & 24.28 & 0.32 & 0.29 \\
\hline $\begin{array}{c}\text { Soft } \\
\text { Thresholding }\end{array}$ & 11.89 & 24.49 & 0.37 & 0.30 \\
\hline $\begin{array}{c}\text { Bayesian } \\
\text { Thresholding }\end{array}$ & 12.27 & 25.03 & 0.48 & 0.30 \\
\hline $\begin{array}{c}\text { FDA Based } \\
\text { Thresholding }\end{array}$ & 12.54 & 25.43 & 0.56 & 0.29 \\
\hline $\begin{array}{c}\text { Proposed } \\
\text { Technique }\end{array}$ & $\mathbf{2 7 . 2 9}$ & $\mathbf{3 4 . 1 6}$ & $\mathbf{0 . 9 1}$ & $\mathbf{0 . 3 2}$ \\
\hline
\end{tabular}

Fig 2 and 3 provide a visual comparison of various speckle filtering approaches, including the proposed technique for reducing speckle noise in Liver and Kidney ultrasound images. Through this investigation, it is closely observed that, the proposed scheme produces the denoised images which is so smoothed and enhanced than other state-of-the-art methods and hence, the denoised images obtained from the suggested technique do not have any checker board or blurring effects in the homogeneous region.

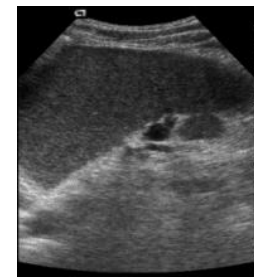

(a)Noisy Image

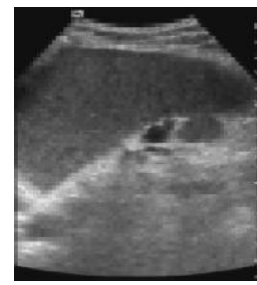

(c) Soft Thresholding

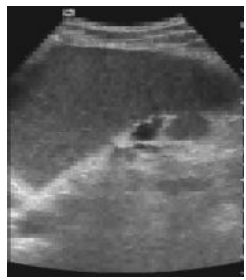

(b) Hard Thresholding

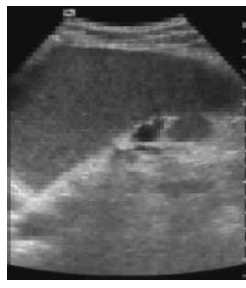

(d) Bayesian Thresholding 


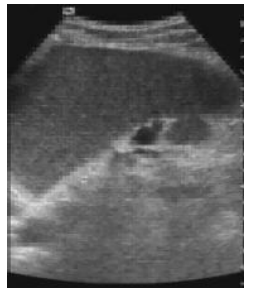

(e) FDA Based

Thresholidng

Fig 2: Visual comparison of the proposed technique including different thresholding approaches for denoising Liver ultrasound image

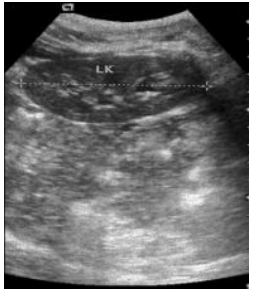

(a) Noisy Image

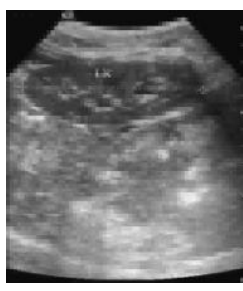

(c) Soft Thresholding

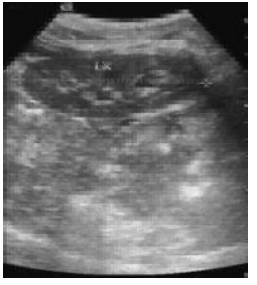

(e) FDA Based

Thresholidng

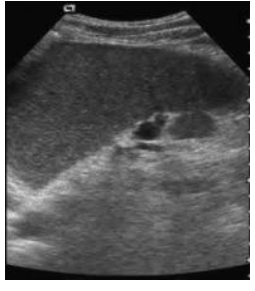

(f) Proposed Technique

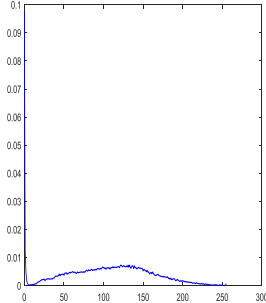

(a) Original Image

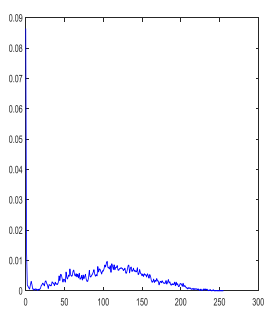

(c) Soft Thresholding

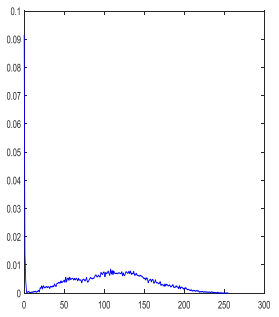

(e) FDA Based

Thresholidng

Fig 4: Probability density curves of original Kidney ultrasound image and denoised images obtained from proposed technique and different thresholding approaches.

\section{CONCLUSION AND FUTURE WORKS}

An effective technique for speckle noise reduction in medical ultrasound images through the wavelet transformation with GA and FDA based threshold parameters have been proposed. In terms of various statistical measurement criteria and visual investigation, the suggested technique shows promising results when compared with various de-speckling method of ultrasound images. According to the discernible evaluation, the denoised image obtained using the proposed technique retains significant details and is free of checkerboard and blurring effects in the homogeneous region, giving physicians and medical experts more competence in recovering critical diagnostic information. As a consequence, the technique is particularly useful for highly inhomogeneous images and can be applied to medical ultrasound images to reduce speckle noise. In future, the more competent speckle noise reduction schemes for ultrasound images will be developed by eminence multi-resolution analysis and adaptive machine learning methods.

\section{REFERENCES}

[1] Achim, A., Bezerianos, A., and Tsakalides, P. 2001. Novel Bayesian multiscale method for speckle removal in medical ultrasound images. IEEE transactions on medical imaging, 20(8), 772-783. 
[2] Donoho, D. L., and Johnstone, I. M. 1995. Adapting to unknown smoothness via wavelet shrinkage. Journal of the american statistical association, 90(432), 1200-1224.

[3] Donoho, D. L., and Johnstone, J. M. 1994. Ideal spatial adaptation by wavelet shrinkage. biometrika, 81(3), 425455.

[4] Donoho, D. L., Johnstone, I. M., Kerkyacharian, G., and Picard, D. $1995 . \quad$ Wavelet shrinkage: asymptopia?. Journal of the Royal Statistical Society: Series B (Methodological), 57(2), 301-337.

[5] Fodor, I. K., and Kamath, C. 2003. Denoising through wavelet shrinkage: an empirical study. Journal of Electronic Imaging, 12(1), 151-160.

[6] Chang, S. G., Yu, B., and Vetterli, M. 2000. Adaptive wavelet thresholding for image denoising and compression. IEEE transactions on image processing, 9(9), 1532-1546.

[7] Chang, S. G., Yu, B., and Vetterli, M. 2000. Spatially adaptive wavelet thresholding with context modeling for image denoising. IEEE Transactions on image Processing, 9(9), 1522-1531.

[8] Chang, S. G., Yu, B., and Vetterli, M. 2000. Wavelet thresholding for multiple noisy image copies. IEEE Transactions on Image Processing, 9(9), 1631-1635.

[9] Jansen, M. 2001. Noise Reduction by Wavelet Thresholding.-Springer Verlag, Lecture notes in Statistics. Vol. 161.

[10] Muhsen, Z. F., Dababneh, M., and Al-Nsour, A. 2011. Wavelet and optimal requantization methodology for lossy fingerprint compression. Int. Arab J. Inf. Technol., 8(4), 383-387.

[11] Rahman, M. M., PK, M. K., and Uddin, M. S. 2014. Optimum Threshold Parameter Estimation of Wavelet Coefficients Using Fisher Discriminant Analysis for Speckle Noise Reduction. International Arab Journal of Information Technology (IAJIT), 11(6).

[12] Mukhopadhyay, S., and Mandal, J. K. 2013. Wavelet based denoising of medical images using sub-band adaptive thresholding through genetic algorithm. Procedia technology, 10, 680-689.

[13] Goldberg, D. E. 1989. Genetic algorithms in search, optimization, and machine learning. Addison. Reading.

[14] Otsu, N. 1979. A threshold selection method from graylevel histograms. IEEE transactions on systems, man, and cybernetics, 9(1), 62-66.

[15] Abo-Eleneen, Z. A. 2011. Thresholding based on Fisher linear discriminant. Journal of pattern recognition research, 2, 326-334.

[16] Sattar, F., Floreby, L., Salomonsson, G., and Lovstrom, B. 1997. Image enhancement based on a nonlinear multiscale method. IEEE transactions on image processing, 6(6), 888-895.

[17] Sivakumar, R., Gayathri, M. K., and Nedumaran, D. 2010. Speckle filtering of ultrasound b-scan images-a comparative study between spatial and diffusion filters. In 2010 IEEE Conference on Open Systems (ICOS 2010) (pp. 80-85). IEEE. 